\title{
Crystallographic Analysis of Transformation Behavior of Acicular Ferrite from B1-type Compounds in Steels
}

\author{
Shoichi NAMBU, ${ }^{1) *}$ Yoshihiko TAKIZAWA, ${ }^{2)}$ Mayumi OJIMA, ${ }^{1)}$ Junya INOUE ${ }^{3)}$ and Toshihiko KOSEKI') \\ 1) Department of Materials Engineering, The University of Tokyo, 7-3-1 Hongo, Bunkyo-ku, Tokyo, $133-8656$ Japan. \\ 2) Department of Materials Engineering. The University of Tokyo. Now at Nomura Research Institute, Ltd., 134 Godo-cho, \\ Hodogaya-ku, Yokohama, 240-0005 Japan. $\quad 3)$ Research Center for Advanced Science and Technology, The University \\ of Tokyo, 4-6-1 Komaba, Meguro-ku, Tokyo, 153-8904 Japan.
}

(Received on January 10, 2017; accepted on March 21, 2017)

\begin{abstract}
Crystallographic analysis was carried out on acicular ferrites (AFs) of steel that nucleated on B1-type compounds embedded in steels during the isothermal transformation from austenite $(\gamma)$ to ferrite $(\alpha)$, and the orientation relationship between $\alpha, \gamma$, and the compounds and the AF formation behavior were correlated by varying the B1-type compound, steel, and transformation temperature. The results demonstrated that all the AFs satisfy the Kurdjumov-Sachs orientation relationship with the prior $\gamma$, and in addition, some of the AFs have specific low-index orientation relationships with the B1-type compounds and nucleate earlier than others. Among the specific low-index orientation relationships, furthermore, AFs satisfying the Baker-Nutting orientation relationship, which gives the lowest interfacial energy between $\alpha$ and a compound, nucleate preferentially.
\end{abstract}

KEY WORDS: acicular ferrite; hetero-nucleation; crystal orientation relationship; transformation.

\section{Introduction}

Intragranular ferrite (IGF), which nucleates at inclusions dispersed in austenite $(\gamma)$ grains, has attracted considerable attention as a means of suppressing the degradation of toughness due to grain-coarsening in the heat-affected zone of steel welds. Thus, many studies have been conducted to clarify the formation mechanisms of IGF, and several factors that promote ferrite $(\alpha)$ nucleation have been reported $^{1-5)}$ such as lattice matching between $\alpha$ and inclusions, thermal stress or strain due to the difference in the thermal expansion between $\gamma$ and inclusions, and changing the chemical composition in the vicinity of the $\gamma /$ inclusion interface.

Regarding lattice matching, B1-type oxides and nitrides (B1) with the $\mathrm{NaCl}$-type lattice structure have been reported to be effective for IGF nucleation ${ }^{6,7)}$ because they have a crystal orientation relationship with low lattice mismatch with $\alpha$, called the Baker-Nutting orientation relationship (B-N OR); $\{100\}_{\mathrm{B} 1} / /\{100\}_{\alpha},<100>_{\mathrm{B} 1} / /<110>_{\alpha}$. However, to rigorously discuss the effect of the lattice coherency on the nucleation of IGF, the change in the $\gamma$-to- $\alpha$ transformation behavior with the transformation temperature should be considered. It is well known that the morphology of IGF is usually divided into two types, polygonal type and acicular type. In the case of polygonal ferrite (PF), ferrites form by a diffusional transformation at relatively high temperatures, and thereby, the B-N OR can be easily satisfied between $\alpha$

\footnotetext{
* Corresponding author: E-mail: nambu@metall.t.u-tokyo.ac.jp DOI: http://dx.doi.org/10.2355/isijinternational.ISIJINT-2017-017
}

and B1 upon the nucleation of $\alpha{ }^{6,7)}$ In the case of acicular ferrite (AF), on the other hand, ferrites form by a displacive transformation at relatively low temperatures and it is well known that a specific crystal orientation relationship exists between the product phase $\alpha$ and the mother phase $\gamma$, which is called the Kurdjumov-Sachs orientation relationship (K-S OR); $\{111\}_{\gamma} / /\{110\}_{\alpha},<110>_{\gamma} / /<111>_{\alpha}$. Under this constraint in the relationship between $\alpha$ and $\gamma$, it is still unclear whether a specific crystal orientation relationship between $\mathrm{AF}$ and $\mathrm{B} 1$ can exist because of the assumption that B1-type compounds do not have a specific orientation relationship with austenite. ${ }^{8)}$ Although several studies have been conducted to consider the crystal orientation relationship between $\alpha, \gamma$, and inclusions, ${ }^{9-12)}$ the kinetics of the IGF nucleation at inclusions, as well as the effect of the crystal orientation relationship between them, has not yet been clarified.

In this study, we focused on the formation behavior of IGF at relatively low temperatures to clarify the crystal orientation relationship between $\alpha, \gamma$, and B1 and its correlation with the transformation behavior of AF. In particular, the selection of the specific crystal orientation relationship between $\alpha$ and B1 was evaluated.

\section{Experimental Procedure}

Two types of steels, C-Mn steel and C-Ni steel, were prepared and their chemical compositions are shown in Table 1. The bainite transformation start temperature (Bs) and martensite transformation start temperature (Ms) calculated using empirical equations ${ }^{13,14)}$ are also shown in Table 1. 
Three different single-crystalline B1-type compounds, TiO, $\mathrm{TiN}$, and $\mathrm{MgO}$, having similar lattice parameters were also prepared. The steels were cut to rod specimens with $6 \mathrm{~mm}$ height and $8 \mathrm{~mm}$ diameter. The B1-type compound particles with diameters from about 3 to $30 \mu \mathrm{m}$ were sandwiched by the two rod specimens, which were then hot-pressed in the height direction to about $50 \%$ reduction at $1100^{\circ} \mathrm{C}$ by a thermomechanical simulator (Thermecmastor-Z, Fuji Electronic Industrial). The specimens were cooled then reheated to $1150^{\circ} \mathrm{C}$ and held for $2400 \mathrm{~s}$ to embed the B1-type compound particles inside the austenite grains as a result of their grain growth. Subsequently, the specimens were cooled rapidly at $50^{\circ} \mathrm{C} \mathrm{s}^{-1}$ by $\mathrm{He}$ gas and held isothermally at 400 , 500 , or $600^{\circ} \mathrm{C}$ to induce the ferrite transformation. Finally, these specimens were gas-quenched to room temperature. A schematic of this thermomechanical cycle is shown in Fig. 1. Note that none of the IGFs nucleated when the specimens were cooled to room temperature at the same cooling rate without isothermal holding.

The heat-treated specimens were cut normal to the interface between the two rod specimens. The observation area was etched by $2 \%$ nital after mechanical polishing and the microstructure was observed by optical microscopy. Additionally, the same area was observed by field-emission scanning electron microscopy (FE-SEM; JSM7001, JEOL) and crystallographic analysis was conducted by electron backscattering diffraction (EBSD; EDAX/TSL) with OIM software (OIM DC and OIM analysis 7). In the crystallographic analysis, not only the B-N OR but also other low-

Table 1. Chemical compositions (in mass \%) of the steels used in this study and their transformation temperatures.

\begin{tabular}{cccccccc}
\hline & $\mathrm{C}$ & $\mathrm{Si}$ & $\mathrm{Mn}$ & $\mathrm{Ni}$ & $\mathrm{Fe}$ & $\mathrm{Bs} /{ }^{\circ} \mathrm{C}$ & $\mathrm{Ms} /{ }^{\circ} \mathrm{C}$ \\
\hline C-Mn steel & 0.35 & 0.20 & 1.76 & - & Bal. & 524 & 337 \\
C-Ni steel & 0.32 & 0.20 & 0.20 & 10.3 & Bal. & 475 & 215 \\
\hline
\end{tabular}

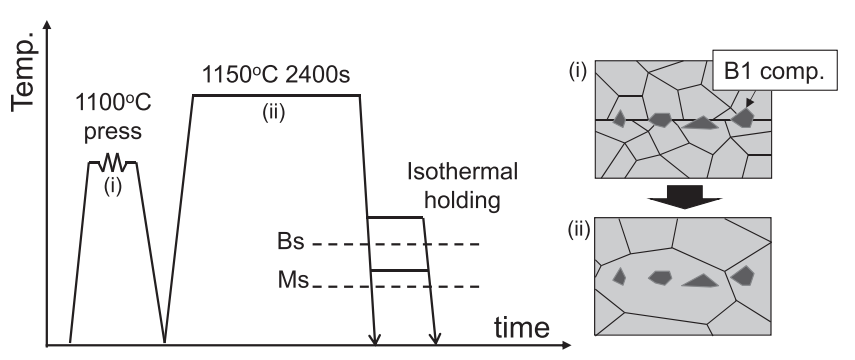

Fig. 1. Thermomechanical cycle used in this study. index ORs such as $\{100\}_{\mathrm{B} 1} / /\{110\}_{\alpha},<110>_{\mathrm{B} 1} / /<110>_{\alpha}$ and $\{110\}_{\mathrm{B} 1} / /\{112\}_{\alpha},<100>_{\mathrm{B} 1} / /<110>_{\alpha}$ (hereafter, called OR2 and OR3, respectively) were also considered because these low-index ORs were also found in our previous studies. $^{7,11)}$

The procedure of evaluating the crystal orientation relationship between $\alpha, \gamma$, and B1 is as follows. The $<100>$ directions of the B1-type compound of interest are set to parallel to RD, TD, and ND in the pole figure. Then specific low-index ORs, the B-N OR, OR2, and OR3, are plotted according to the orientation of the B1-type compound as shown in Fig. 2. In this study, a misorientation within $15^{\circ}$ from the exact orientation relationship is allowed for each specific low-index OR according to our previous study ${ }^{7,11)}$ in which it was found that the misorientation allowance depends on the B1-type compound and the maximum allowance is about $15^{\circ}$ even in the case of a diffusional PF transformation. In this case, the B-N OR and OR3 partially overlap in the pole figure, and the ferrites in the overlapping area, if they exist, are considered to satisfy the B-N OR. Twenty-four variants of the K-S OR in one austenite grain are also plotted in the pole figure using the martensite phase surrounding the B1-type compounds, because the martensite is formed from the remaining austenite by quenching after isothermal holding for a certain period during AF formation. By comparing the experimental results with the above analytical results, the crystal orientation relationship between $\alpha, \gamma$, and B1 is evaluated.

\section{Results and Discussion}

The effect of the transformation temperature on the morphology and the orientation relationship of IGF was investigated using the $\mathrm{C}-\mathrm{Mn}$ steel. The specimens were austenitized at $1150^{\circ} \mathrm{C}$ and then cooled to and isothermally held at 400,500 , or $600^{\circ} \mathrm{C}$ for $10-20 \mathrm{~s}$ as shown in Fig. 1. In the case of the isothermal transformation at $600^{\circ} \mathrm{C}$, only polygonal ferrites (PFs) were observed around the B1-type compounds (TiO) as shown in Fig. 3(a). Both PFs and acicular ferrites (AFs) were observed at $500^{\circ} \mathrm{C}$ (Fig. 3(b)), and only AFs were observed at $400^{\circ} \mathrm{C}$ (Fig. 3(c)). By decreasing the transformation temperature, it was confirmed that the morphology of IGF changed from a polygonal to acicular shape.

The orientation relationship between $\alpha, \gamma$, and B1 was also investigated and the result corresponding to Fig. 3(b) is shown in Fig. 4. The crystal orientation of the $\mathrm{TiO}$ is rotated and its $<100>$ directions are set to parallel to $\mathrm{RD}$,

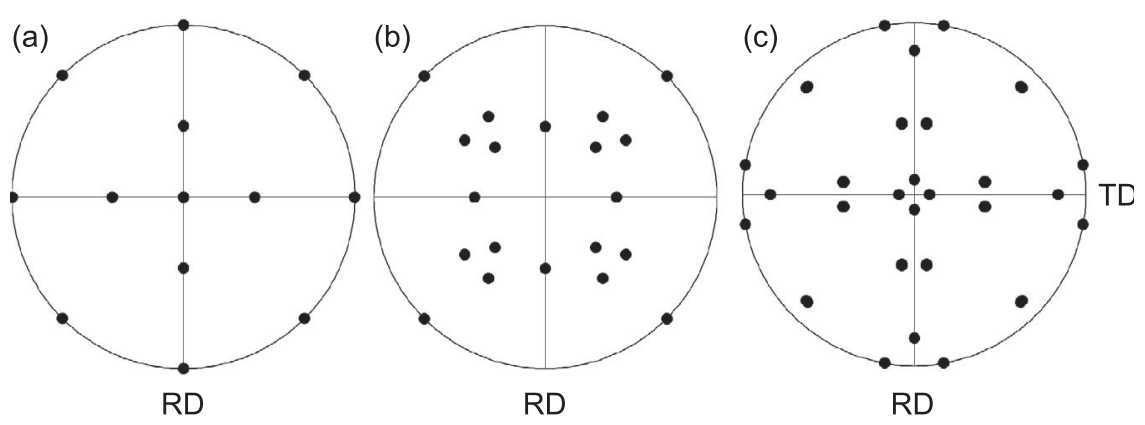

Fig. 2. (001) $\alpha$ pole figures for (a) B-N OR, (b) OR2, and (c) OR3. 

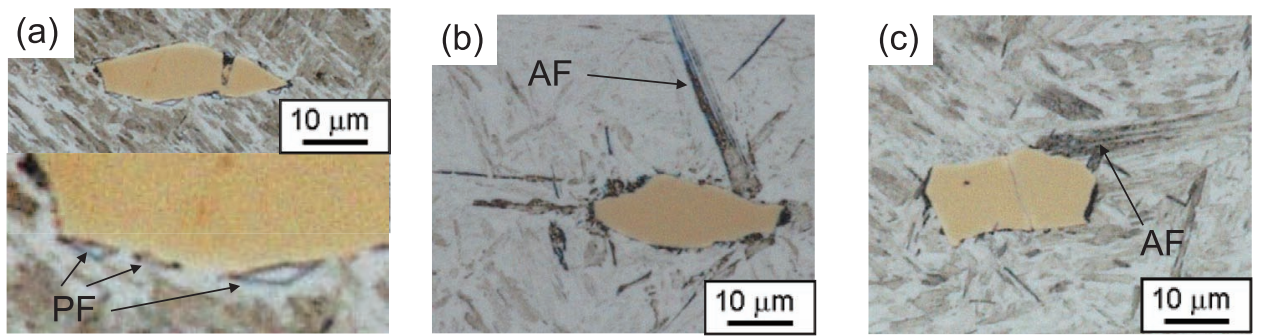

Fig. 3. Optical micrographs of IGFs nucleated on $\mathrm{TiO}$ (a) after $20 \mathrm{~s}$ at $600^{\circ} \mathrm{C}$, (b) after $10 \mathrm{~s}$ at $500^{\circ} \mathrm{C}$, and (c) after $10 \mathrm{~s}$ at $400^{\circ} \mathrm{C}$.
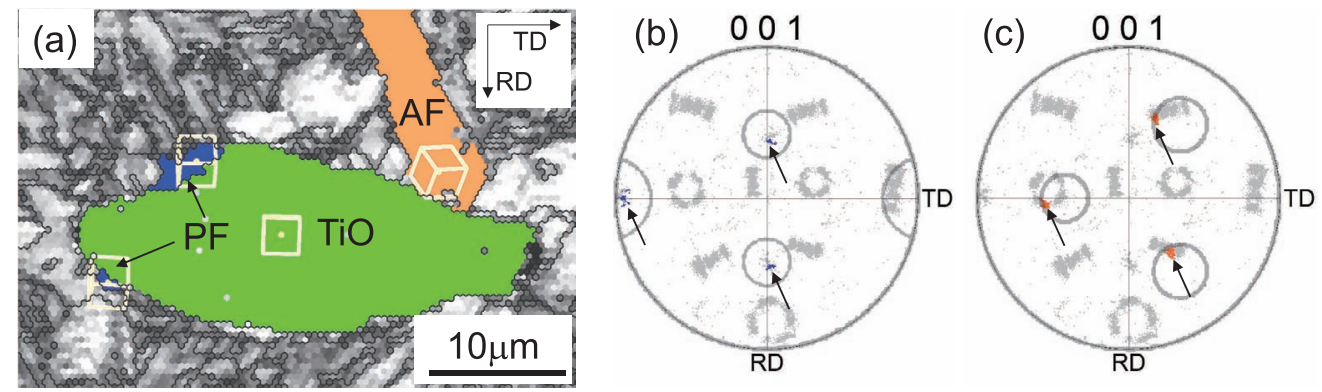

Fig. 4. Crystal orientation analysis for $\mathrm{PFs}$ and $\mathrm{AF}$ nucleated on $\mathrm{TiO}$ after $10 \mathrm{~s}$ at $500^{\circ} \mathrm{C}$ (corresponding to Fig. 3(b)). (a) Image quality map with highlighted TiO, PFs, and AF. (001) $\alpha$ pole figures for (b) PFs with a circle having $15^{\circ}$ misorientation from the B-N OR and (c) AF with a circle having $15^{\circ}$ misorientation from OR2.
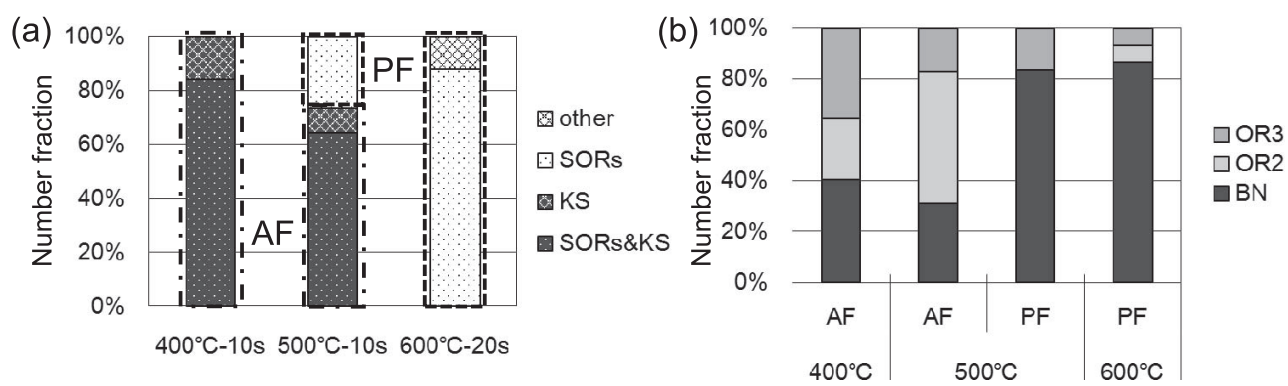

Fig. 5. Fractions of IGFs categorized on the basis of the crystal orientation relationship between $\alpha, \gamma$, and $\mathrm{B} 1$ generated at 400,500 , and $600^{\circ} \mathrm{C}$.

TD, and ND as shown in Fig. 4(a). The crystal orientations of the PFs specified in Fig. 4(a) are plotted in the circle which is centered at the B-N OR and has a $15^{\circ}$ radius, but away from the K-S OR (Fig. 4(b)). The crystal orientation of the AF specified in Fig. 4(a) is plotted in the circle which is centered at OR2 and has a $15^{\circ}$ radius, and also placed in the K-S OR (Fig. 4(c)). The result demonstrates that the PFs satisfy the B-N OR with TiO but not with the prior $\gamma$, and the AF satisfies OR2 with $\mathrm{TiO}$ and the K-S OR with prior $\gamma$ simultaneously in the case of Fig. 4. The orientation relationship between $\alpha, \gamma$, and B1 was evaluated for about 50 IGF grains nucleated at $\mathrm{TiO}$ at each isothermal transformation temperature, and the fractions of the orientation relationships are shown in Fig. 5. In Fig. 5(a), the orientation relationships are categorized into four types, where "SORs" means that the IGF satisfies one of the specific lowindex ORs with the compound (B-N OR, OR2, and OR3) but not with the prior $\gamma$, "KS" means that the IGF satisfies the K-S OR with the prior $\gamma$ but not with the compound, "SORs\&KS" means that the IGF satisfies both the K-S OR with the prior $\gamma$ and one of the specific low-index ORs with the compound, and "other" means that no specific low-index
ORs were found between $\alpha, \gamma$, and B1. From the results for 500 and $600^{\circ} \mathrm{C}$, most of the PFs, delineated by the dashed line in the figure, have specific low-index ORs with the compounds. The results for 400 and $500^{\circ} \mathrm{C}$ show that every $\mathrm{AF}$, delineated by the chain line in the figure, satisfies the K-S OR with the prior $\gamma$, and many of the AFs also satisfy specific low-index ORs with the compounds. Details of the SORs between $\alpha$ and B1 are summarized in Fig. 5(b). In the case of PF, the fraction of IGFs satisfying the B-N OR with the compound is more than $80 \%$. This result clearly demonstrates that the B-N OR is preferentially selected during the diffusional transformation from $\gamma$ to PF. On the other hand, in the case of $\mathrm{AF}$, the fraction of $\mathrm{BN}$ is smaller but the fraction of other low-index ORs, OR2 and OR3, is larger. Thus, a clear difference between PF and AF in terms of the crystal orientation relationship with the compound was found; in the case of PF, the B-N OR dominates, while in the case of AF, not only the B-N OR but also other lowindex ORs, OR2 and OR3, are selected.

The change in the selection of the orientation relationships between $\alpha$ and $\mathrm{B} 1$ during the isothermal transformation of $\mathrm{AF}$ at $400^{\circ} \mathrm{C}$ with time was evaluated using combinations 


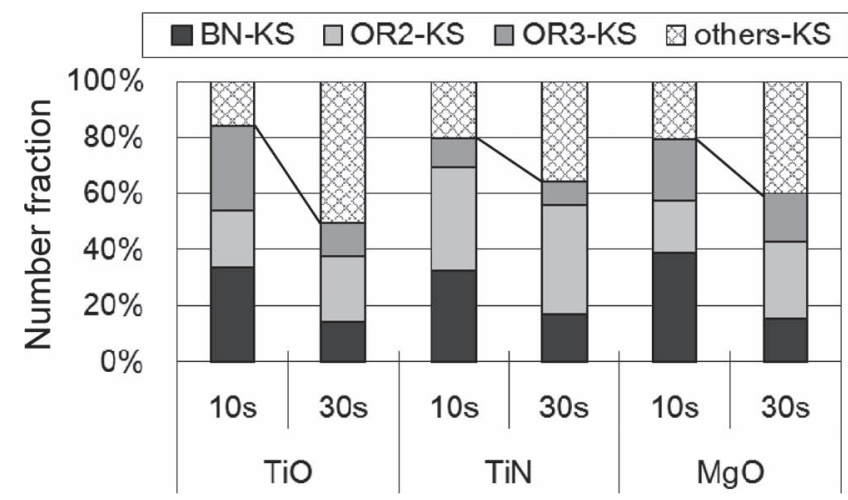

Fig. 6. Change in the fraction of IGFs satisfying a specific orientation relationship with the B1-type compounds, TiO, TiN, and $\mathrm{MgO}$ with the holding time at $400^{\circ} \mathrm{C}$.

of the C-Mn steel and different B1-type compounds, and the results are shown in Fig. 6. For every condition, about 50-70 IGF grains were analyzed. From the result using $\mathrm{TiO}$, in the early stage of the transformation such as after $10 \mathrm{~s}$ of isothermal holding, more than $80 \%$ of IGFs satisfy the low-index ORs with the compound as well as the K-S OR with the prior $\gamma$, while the fraction of IGFs that do not satisfy any low-index ORs with the compound becomes large with increasing holding time up to $30 \mathrm{~s}$. In the cases of $\mathrm{TiN}$ and $\mathrm{MgO}$, the fraction of IGFs satisfying the low-index ORs with the compound is relatively large in the early stage of the transformation and becomes small with time. These results suggest that IGFs having specific crystal orientation relationships with both the prior $\gamma$ and the compound form first, and then IGFs only satisfying the K-S OR with the prior $\gamma$ start to form after a relatively long incubation time.

To further investigate the selection mechanism of the specific low-index ORs (B-N OR, OR2, and OR3) between $\mathrm{AF}$ and $\mathrm{B} 1$, the $\mathrm{C}-\mathrm{Ni}$ steel was subjected to the same experiments as the $\mathrm{C}-\mathrm{Mn}$ steel, because the driving force of the $\gamma$-to- $\alpha$ transformation in $\mathrm{C}-\mathrm{Ni}$ steel at a given temperature is always smaller than that in $\mathrm{C}-\mathrm{Mn}$ steel at the same temperature and the selection of the coherent interface between $\alpha$ and B1 is affected by the driving force. It is assumed that a coherent interface with smaller interfacial energy is more likely selected when the driving force is small. Figure 7 shows the change in the fractions of the orientation relationships for AFs that nucleate at TiN during isothermal holding at $400^{\circ} \mathrm{C}$. At the beginning of AF formation, at 7200 $\mathrm{s}$ in this case, more than $80 \%$ of AFs satisfy the specific low-index ORs, and the fraction satisfying the B-N OR is the largest among the low-index ORs. Subsequently, the fractions satisfying OR2 and OR3 increase with time, and finally the fraction satisfying other relationships becomes the largest. This result also suggests that the B-N OR was selected preferentially at the beginning of the transformation, then other low-index ORs were also selected with increasing time, and finally more random ORs became dominant rather than low-index ORs such as the B-N OR, OR2, and OR3. Even in the formation of AFs satisfying the K-S OR with the prior $\gamma$, it appears that AFs having lower interfacial energy between $\alpha$ and B1 can nucleate with a shorter incubation time on the B1-type compounds.

When AFs nucleate on B1-type compounds with specific

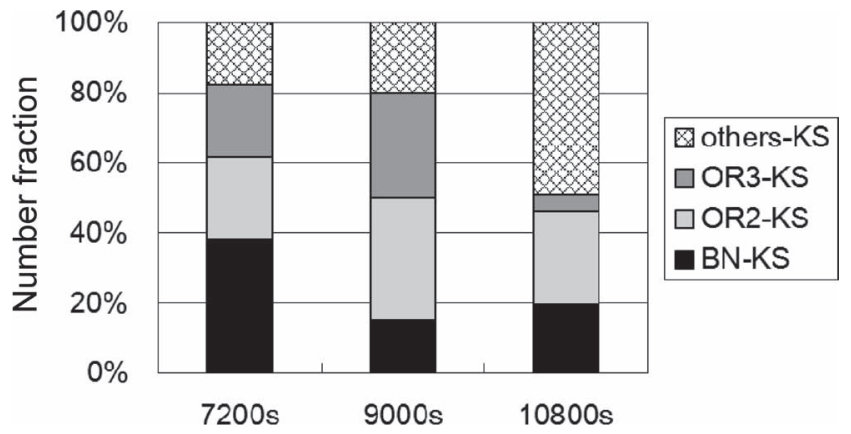

Fig. 7. Change in the fraction of IGFs satisfying the specific orientation relationship with TiN during isothermal holding at $400^{\circ} \mathrm{C}$.

ORs, they must satisfy the K-S OR with the prior $\gamma$ simultaneously. The probability that AF simultaneously satisfies the specific ORs with the compounds and the K-S OR with the prior $\gamma$ was calculated using the Euler space assuming that $\mathrm{B} 1$ and $\gamma$ are randomly oriented. The matrix representing the rotation given by the Euler angle (Bunge notation), $g=\left(\varphi_{1}, \phi, \varphi_{2}\right)$, can be given as

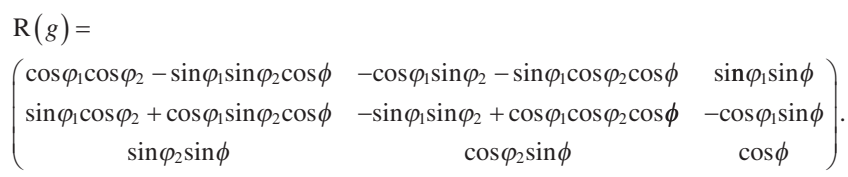

Firstly, the $<001>$ direction of the B1-type compound is fixed at a Euler angle $g_{0}=(0,0,0)$ as a reference. The rotation of $\alpha$ satisfying the specific ORs, the B-N OR, OR2, and OR3, with this B1-type compound can be represented by the following equation:

$$
\Delta g_{\alpha}^{O R} \equiv\left(\varphi_{1}, \phi, \varphi_{2}\right)= \begin{cases}(45,0,0) & : \mathrm{B}-\mathrm{N} \\ (45,90,45) & : \text { OR2 } \ldots \ldots \ldots . . . \\ (170,90,135) & : \text { OR3 }\end{cases}
$$

In addition, the symmetry due to the cubic structure should be considered. In the case of a cubic structure, there are 24 combinations of planes and directions as shown in Ref. 15). The Euler angle of $\alpha$ satisfying specific ORs with a compound including the symmetry can be given by

$$
R\left(g_{\alpha}\right)=S_{i} \cdot R\left(\Delta g_{\alpha}^{O R}\right) \cdot S_{j} \cdot R\left(g_{0}\right)=S_{i} \cdot R\left(\Delta g_{\alpha}^{O R}\right) \cdot S_{j} \cdot E,
$$

where $S_{i}$ is the rotation of symmetry and $E$ is the identity matrix. The Euler angle for the rotation of the K-S OR is given by ${ }^{16)}$

$$
\Delta g_{K S} \equiv\left(\Delta \varphi_{1}, \Delta \phi, \Delta \varphi_{2}\right)=(5.77,48.19,5.77) .
$$

Regarding this rotation, the symmetry of the cubic structure also has to be taken into account. Therefore, the Euler angle of $\gamma$ satisfying the K-S OR with $\alpha$ is represented by

$$
R\left(g_{\gamma}\right)=S_{i} \cdot R\left(\Delta g_{K S}\right) \cdot S_{j} \cdot R\left(g_{\alpha}\right) .
$$

From Eqs. (3) and (5), the Euler angle of $\gamma$ satisfying the K-S OR with $\alpha$ that can satisfy specific orientation relationships is obtained as 


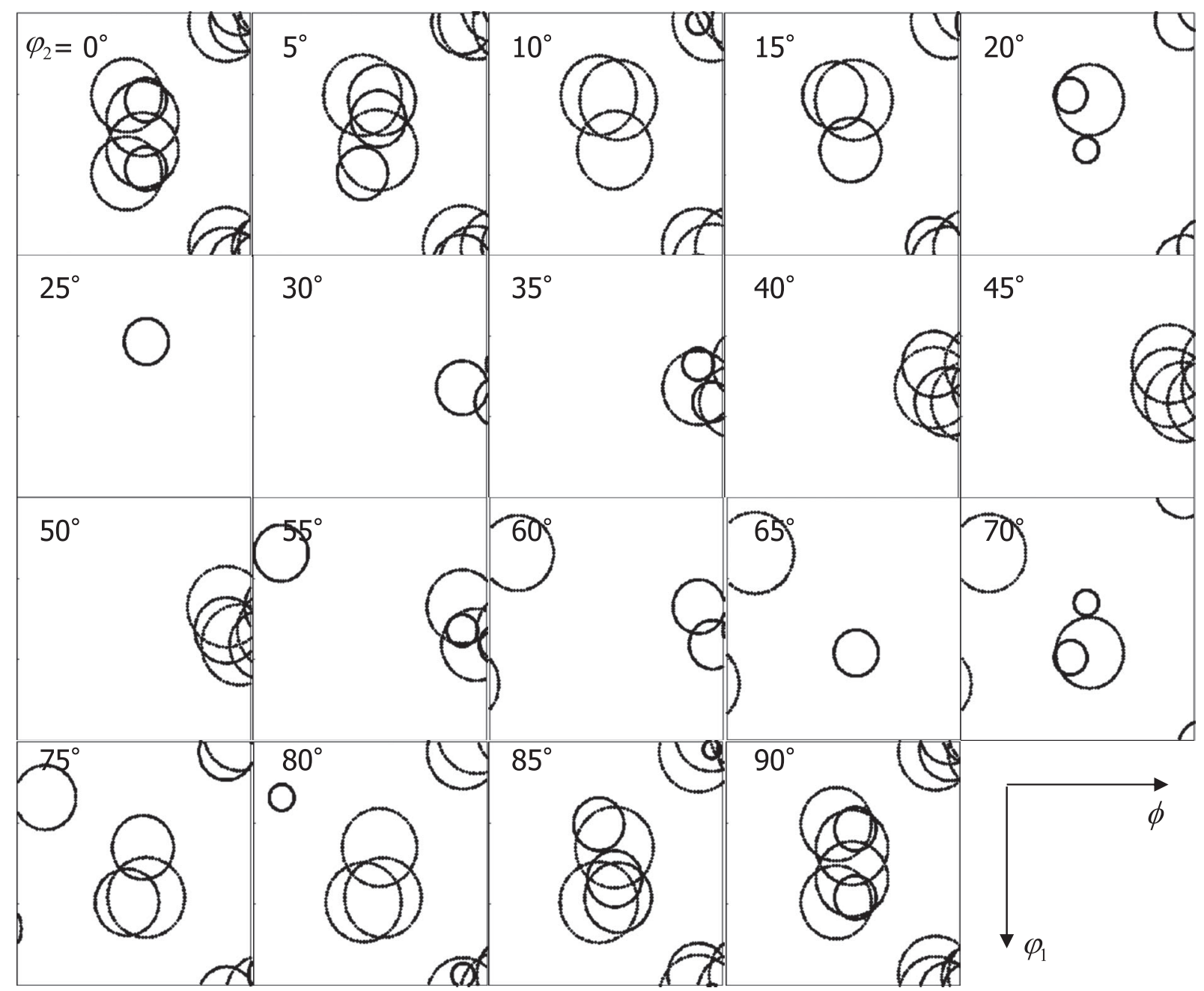

Fig. 8. Plane map of Euler space obtained by the calculation in the case of the B-N OR.

$$
R\left(g_{\gamma / \alpha / B 1}\right)=S_{i} \cdot R\left(\Delta g_{K S}\right) \cdot S_{j} \cdot R\left(\Delta g_{\alpha}^{O R}\right) \cdot S_{k} \cdot E .
$$

Since a misorientation within $15^{\circ}$ from the exact orientation relationship is allowed when the crystal orientation relationship between $\alpha$ and B1 is evaluated, the fraction of the sphere that is centered at the Euler angle obtained by Eq. (6) and has a $15^{\circ}$ radius in the Euler space was calculated to evaluate the probability. In this calculation, the overlapping space between the B-N OR and OR3 is considered to be the B-N OR as well as the experimental analysis. Figure 8 shows the calculation result of Euler space with the spheres obtained when the ferrite simultaneously satisfies the K-S OR with the prior $\gamma$ and the B-N OR with the B1-type compound. In this figure, there are some overlapping spaces, but they are not counted more than once in the calculation. Based on the above calculation, the probability that the ferrite simultaneously satisfies both the K-S OR and the B-N OR is about $18.4 \%$ when a B1-type compound exists in an austenite grain with a random orientation. The probabilities for the cases of OR2 and OR3 are 19.9\% and 9.5\%, respectively. Each probability is shown in Fig. 9. The ferrites, which are categorized as others with the fraction of $52.2 \%$, satisfy the K-S OR with the prior $\gamma$ while they nucleate without any specific ORs with B1-type compound. On the other hand, the fractions of ferrites that satisfy the specific ORs with both $\gamma$ and the B1-type compound in the

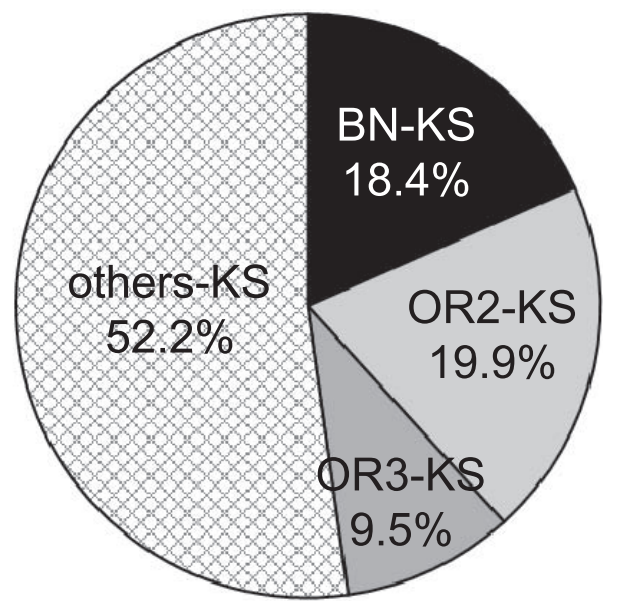

Fig. 9. Probabilities that specific orientation relationships are satisfied between $\alpha, \gamma$, and B1.

early stage of the transformation, obtained by the experiment and shown in Figs. 6 and 7, are larger than the probabilities obtained by the calculation and shown in Fig. 9, and therefore the fractions obtained by experiments do not result from stochastic nucleation events but from nucleation with low-index planes having low interfacial energies, even in the case of a displacive AF transformation.

From the results, it was found that AFs, which nucleate at a relatively low temperature and have to satisfy the K-S 
OR with the prior $\gamma$, can nucleate without any specific ORs with B1-type compound probably due to the large driving force of transformation. However, we assume that ferrites satisfying the K-S OR with the prior $\gamma$ and the B-N OR with the compound nucleate preferentially because they have the lowest interfacial energy between $\alpha$ and $\gamma$ and between $\alpha$ and B1; such nucleation sites where the specific relationships between $\alpha, \gamma$, and $\mathrm{B} 1$ are satisfied are selected first. Subsequently, those satisfying the K-S OR and other low-index orientation relationships, OR2 and OR3, nucleate, and as time proceeds, those satisfying the K-S OR with the prior $\gamma$ but no specific orientation relationships with the compound start to nucleate at the remaining sites of the compound.

\section{Conclusions}

(1) It was confirmed that the Baker-Nutting orientation relationship between ferrites and B1-type compounds is preferentially selected in the case of polygonal ferrites, which nucleate at a relatively high temperature.

(2) In the case of acicular ferrites, which nucleate at a relatively low temperature, ferrites satisfying the BakerNutting orientation relationship between $\alpha$ and B1 nucleate preferentially in spite of the constraint imposed by the Kurdjumov-Sachs orientation relationship between $\alpha$ and $\gamma$ in the early stage of the transformation.

(3) Acicular ferrites having other orientation relationships also nucleate as the transformation proceeds.

(4) In the early stage of the transformation, the fraction of acicular ferrites satisfying both the Kurdjumov-Sachs orientation relationship with the prior austenite and specific crystal orientation relationships with the compound obtained by experiments is higher than that calculated from the Euler space assuming stochastic nucleation events. Nucleation with low-index planes having low interfacial energies takes place intensively in the early stage of the transformation even in the displacive acicular ferrite transformation.

\section{Acknowledgement}

This work was supported by ISIJ Research Promotion Grant.

\section{REFERENCES}

1) S. S. Babu: Curr. Opin. Solid State Mater. Sci., 8 (2004), 267.

2) T. Koseki and G. Thewlis: Mater. Sci. Technol., 21 (2005), 867.

3) J. M. Gregg and H. K. D. H. Bhadeshia: Acta Metall. Mater., 42 (1994), 3321.

4) S. Zhang, N. Hattori, M. Enomoto and T. Tarui: ISIJ Int., 36 (1996), 1301.

5) Y. Kang, S. Jeong, J.-H. Kang and C. Lee: Metall. Mater. Trans. A, 47A (2016), 2842.

6) T. Furuhara, T. Shinyoshi, G. Miyamoto, J. Yamaguchi, N. Sugita, N. Kimura, N. Takemura and T. Maki: ISIJ Int., 43 (2003), 2028.

7) K. Kasai, C. J. Lee, S. Nambu, J. Inoue and T. Koseki: Tetsu-toHagané, 96 (2010), 123.

8) H. K. D. H. Bhadeshia: Bainite in Steels, 3rd Ed., Maney Publishing, Leeds, (2015), 258.

9) Ø. Grong, A. O. Kluken, H. K. Nylund, A. L. Dons and J. Hjelen: Metall. Mater. Trans. A, 26A (1995), 525.

10) L. Cheng and K. M. Wu: Acta Mater., 57 (2009), 3754.

11) C. J. Lee, S. Nambu, J. Inoue and T. Koseki: ISIJ Int., 51 (2011), 2036.

12) H. Nako, H. Hatano, Y. Okazaki, K. Yamashita and M. Otsu: ISIJ Int., 54 (2014), 1690.

13) R. L. Bodnar, T. Ohhashi and R. I. Jaffee: Metall. Trans. A, 20A (1989), 1445.

14) K. W. Andrews: J. Iron Steel Inst., 203 (1965), 721.

15) M. Humbert, F. Wagner and C. Esling: J. Appl. Crystallogr., 25 (1992), 724.

16) G. Nolze: Cryst. Res. Technol., 41 (2006), 72. 